\title{
Enhancement of Edge-based Image Quality Measures Using Entropy for Histogram Equalization-based Contrast Enhancement Techniques
}

\author{
Hasan Thabit Rashid Kurmasha \\ Computer Department \\ College of Education for Girls \\ Kufa University \\ Najaf, Iraq \\ Hasant.kurmasha@uokufa.edu.iq \\ Chen Soong Der \\ Department of Graphics and Multimedia \\ College of Information Technology \\ University Tenaga Nasional \\ Selangor D.E., Malaysia \\ ChenSoong@uniten.edu.my
}

\author{
Abbas Fadhil Hamzah Alharan \\ Computer Department \\ College of Education for Girls \\ Kufa University \\ Najaf, Iraq \\ abbasf.abood@uokufa.edu.iq
}

\author{
Nur Hanani Azami \\ Department of Graphics and Multimedia \\ College of Information Technology \\ University Tenaga Nasional \\ Selangor D.E., Malaysia \\ trulyhanani@gmail.com
}

\begin{abstract}
An Edge-based image quality measure (IQM) technique for the assessment of histogram equalization (HE)based contrast enhancement techniques has been proposed that outperforms the Absolute Mean Brightness Error (AMBE) and Entropy which are the most commonly used IQMs to evaluate Histogram Equalization based techniques, and also the two prominent fidelity-based IQMs which are Multi-Scale Structural Similarity (MSSIM) and Information Fidelity Criterion-based (IFC) measures. The statistical evaluation results show that the Edge-based IQM, which was designed for detecting noise artifacts distortion, has a Person Correlation Coefficient (PCC) > 0.86 while the others have poor or fair correlation to human opinion, considering the Human Visual Perception (HVP). Based on HVP, this paper propose an enhancement to classic Edgebased IQM by taking into account the brightness saturation distortion which is the most prominent distortion in HE-based contrast enhancement techniques. It is tested and found to have significantly well correlation (PCC $>0.87$, Spearman rank order correlation coefficient $($ SROCC) $>\mathbf{0 . 9 2}$, Root Mean Squared Error $($ RMSE) $<0.1054$, and Outlier Ratio $(\mathrm{OR})=0 \%$ ).
\end{abstract}

Keywords-Histogram Equalization; Contrast enhancement; Image Quality measures; Distortions; Human Visual perception

\section{INTRODUCTION}

Automatic assessment of image quality is the target of an Image Quality Assessment (IQA) algorithm, therefore a great deal of effort has been made to develop IQA algorithms that correlate well with Human Visual Perception (HVP) of distortions. Absolute Mean Brightness Error (AMBE) and Entropy are two popular IQA algorithms for assessment of Histogram Equalization (HE) based contrast enhancement method. HE technique is widely used in various fields however, HE could cause annoying distortions such as excessive brightness change, noise artifacts and brightness saturation (see Figure 1) and because of this it is not commonly used in consumer electronic devices. Hence, many modifications of HE-based techniques have been suggested over the years. They can be distinguished in two main classes. Automatic (the processing does not need user interference [112]) and adjustable (the user needs to adjust the parameters to control the degree of enhancement). It is important for the IQM to have a good correlation with HVP. Therefore, this subjective evaluation investigates the problem of noise artifacts distortion in HE-based technique from perspective of the IQM's correlation with human opinion on the perceived annoyance of distortions in relating with the luminance and texture masking of HVP [13]. This paper concentrates on the problem of the second type of distortions (brightness saturation) in automatic HE-based techniques. The paper is organized as follows: In Section II a review of basic IQMs is presented. The proposed IQM framework and the algorithm details are presented in Section III. The evaluation and discussion of the work and results related to HVP in Section IV. The conclusion and recommended future work in Section $\mathrm{V}$.

\section{REVIEW OF IQMS}

\section{A. $A M B E$}

AMBE is the absolute difference between the mean of input and output image and basically defined by $(\mathrm{AMBE}=\mid \mathrm{E}(\mathrm{X})-$ $\mathrm{E}(\mathrm{Y}) \mid)$ Where, $\mathrm{X}$ and $\mathrm{Y}$ denote the input and output image 
respectively, and $\mathrm{E}(\cdot)$ denotes the expected value, i.e. the statistical mean. AMBE is designed to detect the excessive brightness change distortion. Noise artifacts and brightness saturation distortions are not taken into account by AMBE, hence using it to detect other types of distortion could be misleading as explained in Figure 2.

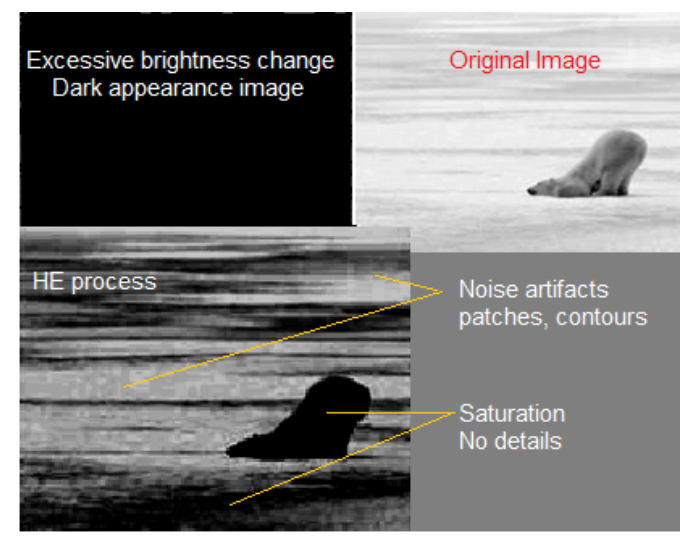

Fig. 1. HE's three distorstions types.

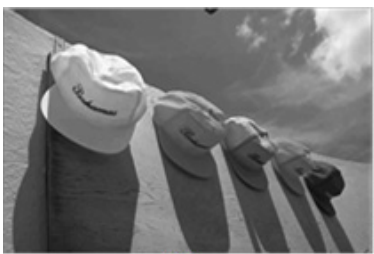

(A)

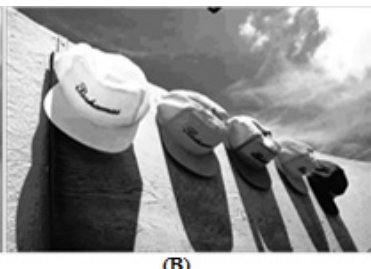

(B)
Fig. 2. Caps image with two different AMBE values, (B) caps with less AMBE (14.02) obviously shows the noise distortion in the background and brightness saturation in the image which is not seen in (A) with high AMBE (37.31).

\section{B. Entropy Measure}

Entropy is a measure of the uncertainty associated with a random variable (Shannon Entropy). It counts, in the sense of an expected value, the information contained in an information source (the image). Hypothetically, higher entropy means more information is available from the information source. Therefore, an image having higher entropy is considered to have a better quality [14]. Since the global gray levels have relatively low probability density, HE tends to combine them and results in decrease of entropy although such action tends to increase the contrast of an image as we see in Figure 3.

\section{Edge-based IQM}

In general, the noise artifacts distortions appear in the form of edges found in distorted images generated by HE-based techniques. They however are not found in the original one. Thus, edge detection is the basic step to extract the features of the image annoying distortion in this metric. Basically, image annoying distortion can be classified as noise artifacts when the edge points are noticed in distortion image and not noticed in the original image. HVP masking incorporates with the detection of noise artifacts [13]. This paper proposes to develop this IQM by taking into account the second most popular type of distortion which is the brightness saturation incorporating with HVP.

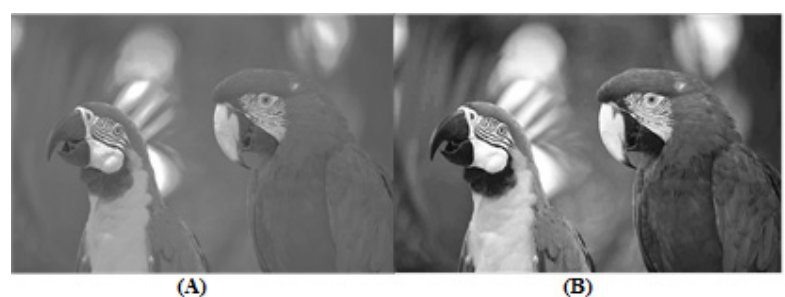

Fig. 3. Parrots image with two different entropy values, (B) parrots with lower entropy (5.72) clearly shows better contrast than (A) with higher entropy (5.74).

\section{PROPOSED IQM}

The generic framework of the proposed Entropy-based IQM is developed as shown in Figure 4.

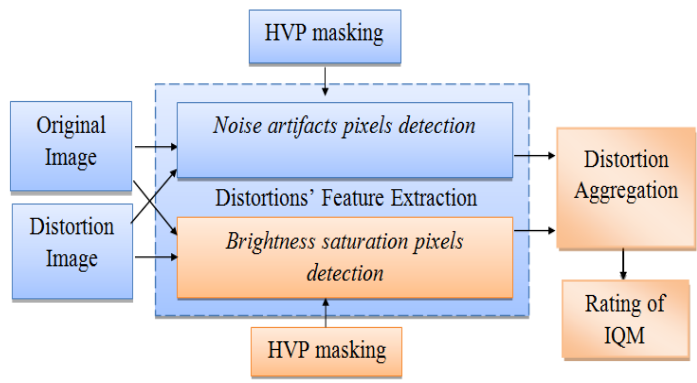

Fig. 4. Generic framework of proposed IQM.

\section{A. Proposed IQM Framework}

1. Brightness saturation's Features collection: This work's purpose is to develop the edge-based IQM (noise detection) by concentrating on the brightness saturation which is commonly found in the output images of HE-based techniques. In HE-based technique, the brightness saturation occurs when there are very obvious and annoying noise artifacts. This is due to the contrast stretching mechanism of $\mathrm{HE}$ which performs more contrast stretching to dominant gray levels which in turn may lead to the extension of noise artifacts, while contrast shrinks those which may cause brightness saturation [13]. Hence, brightness saturation happens when several gray levels are grouped into one. Therefore, it can be detected as a decline in the entropy of gray level and to measure that, HVP features were taken into account. The proposed enhancement will be further elaborated into the next subsection

2. Algorithms Gathering: To get two types of annoying distortion, all detected pixels of noise artifacts from noise algorithm and pixels of brightness saturation from brightness saturation algorithm are gathered according to their respective weight in a single IQM. 


\section{B. Generic Proposed IQM Algorithm}

The general flowchart of the proposed Entropy-based IQM algorithm is shown in Figure 5.

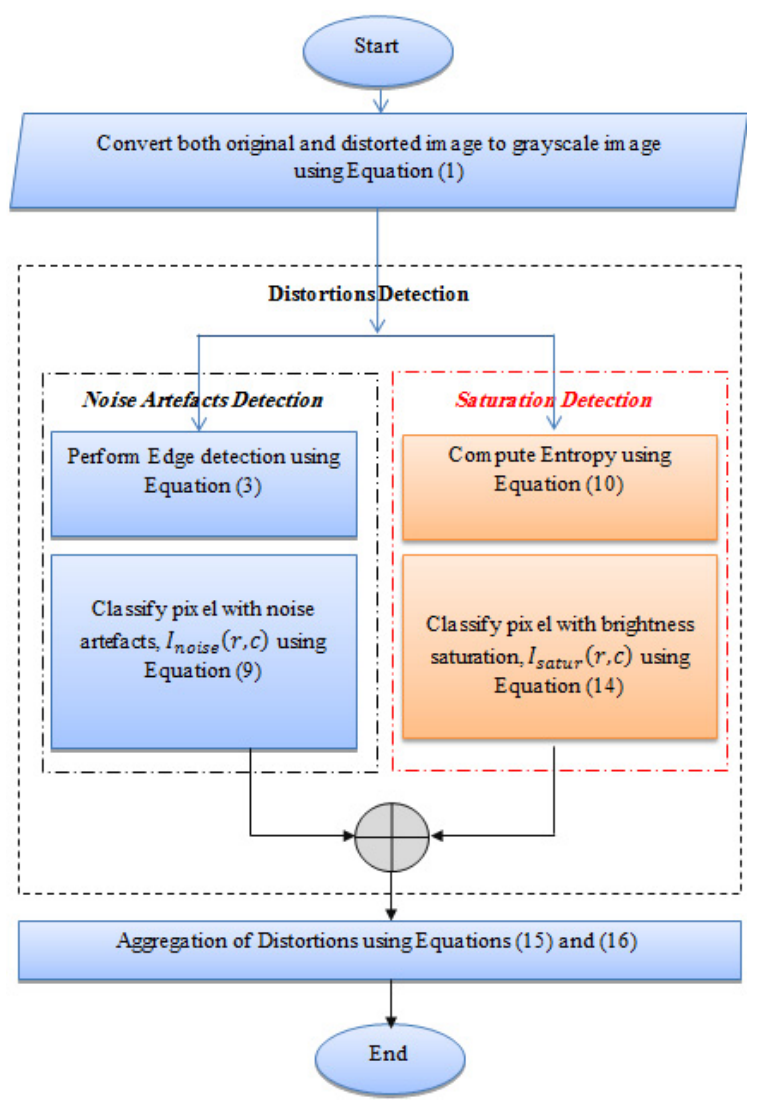

Fig. 5. Flowchart of the proposed IQM algorithm.

Prior to edge detection, the colour image is converted to grayscale image using the conversion in (1):

$\mathrm{I}_{\text {gray }}(\mathrm{r}, \mathrm{c})=0.2989 \mathrm{I}_{\text {red }}(\mathrm{r}, \mathrm{c})+0.5870 \mathrm{I}_{\text {green }}(\mathrm{r}, \mathrm{c})+0.1140 \mathrm{I}_{\text {blue }}(\mathrm{r}, \mathrm{c})(1)$

Edges are detected by using the Sobel operator which has two masks, the horizontal mask (row mask) $\mathrm{M}_{\mathrm{h}}$, and the vertical mask (column mask) $\mathrm{M}_{\mathrm{v}}$, defined as:

$$
M_{h}=\frac{1}{8}\left[\begin{array}{ccc}
-1 & -2 & -1 \\
0 & 0 & 0 \\
1 & 2 & 1
\end{array}\right], \quad M_{v}=\frac{1}{8}\left[\begin{array}{ccc}
-1 & 0 & 1 \\
-2 & 0 & 2 \\
-1 & 0 & 1
\end{array}\right]
$$

The edge magnitude of a point at row $r$ and column $\mathrm{c}$ of an image is defined as below.

$$
\begin{aligned}
& \operatorname{EM}(r, c)=\sqrt{S_{r}^{2}+S_{c}^{2}} \\
& S_{r}=\sum_{i=0}^{n-1} \sum_{j=0}^{m-1} I(r-1+i, c-1+j) M_{h}(i, j)
\end{aligned}
$$

$$
S_{c}=\sum_{i=0}^{n-1} \sum_{j=0}^{m-1} I(r-1+i, c-1+j) M_{v}(i, j)
$$

In a distorted image, if a point's edge magnitude is equal or exceeds its predetermined threshold value while the edge magnitude of the same point in the original image is less than the predetermined threshold value then the point is classified as edge point. The threshold value Thr_Image Edge (r, c) for both images is counted based on the effect of luminance masking by considering two thresholds (Thr Low Lum, and Thr_High_Lum) to compare with the luminance level average Lum_Avg $(r, c)$ of each sub- image $n \times n(n=3)$. The suggested standard threshold value $\mathrm{T}$ is chosen to be the standard threshold which is used for normalization of both the original and distorted images $\mathrm{I}(\mathrm{r}, \mathrm{c})$ as shown below.

$$
\left.\begin{array}{l}
\text { Thr_Image_Edge }(\mathrm{r}, \mathrm{c})=\mathrm{T}^{2}, \\
\text { If }\left(\operatorname{Lum} m_{-} \operatorname{Avg}(r, c) \geq T h r_{-} L o w_{-} \text {Lum }\right) \\
\text { And }\left(\operatorname{Lum}_{-} \operatorname{Avg}(r, c) \leq T h r_{-} H_{\text {High_Lum }}\right)
\end{array}\right\}
$$

Or:

$$
\left.\begin{array}{c}
\text { Thr_Image_Edge }(\mathrm{r}, \mathrm{c})=2 \mathrm{~T}^{2}, \\
\text { If }\left(\text { Lum_Avg }(r, c)<T h r_{-} \text {Low_Lum }\right) \\
\text { Or }\left(\operatorname{Lum} m_{-} \operatorname{Avg}(r, c)>T h r_{-} \text {High_Lum }_{-}\right.
\end{array}\right\}
$$

A point is classified as noise if there is edge detected in distorted image $\left(\mathrm{EM}_{\mathrm{D}}(\mathrm{r}, \mathrm{c}) \geq \mathrm{Thr}\right.$ Image_Edge $\left.(\mathrm{r}, \mathrm{c})\right)$ but not detected in original image $\left(\mathrm{EM}_{\mathrm{O}}(\mathrm{r}, \mathrm{c})<\mathrm{Thr}\right.$ Image_Edge $(\mathrm{r}, \mathrm{c})$ where $\operatorname{EM}_{\mathrm{D}}(\mathrm{r}, \mathrm{c})$ and $\mathrm{EM}_{\mathrm{O}}(\mathrm{r}, \mathrm{c})$ denote the edge magnitudes of distorted and original image respectively. In order to acquire the effect of texture masking for the original image, the activity of sub-image is computed by the local entropy Entropy(r, c) where low entropy indicates low level of activity. Only noise in sub-image with activity level less than the suggested predetermined threshold Thr_Noise_Entropy is considered to be visible noise artifacts.

$$
I_{n o i s e}(r, c)=\left\{\begin{aligned}
1, & \text { If }\left(\mathrm{EM}_{\mathrm{O}}(\mathrm{r}, \mathrm{c})<\mathrm{Thr}\right. \text { IImage_Edge(r,c)) } \\
& \text { And }\left(\mathrm{EM}_{\mathrm{D}}(\mathrm{r}, \mathrm{c}) \geq \mathrm{Thr} \_ \text {Image_Edge(r,c) }\right) \\
& \text { And }\left(\operatorname{Entropy}_{O}(\mathrm{r}, \mathrm{c})<\mathrm{Thr} \text { Noise_Entropy }\right) \\
0, & \text { Else }
\end{aligned}\right.
$$

$$
\operatorname{Entropy}(r, c)=-\sum_{g=0}^{l-1} P_{l}(g) \log 2 P_{l}(g)
$$

$\mathrm{P}_{1}(\mathrm{~g})$, means the probability of gray level $\mathrm{g}$ which is the total number of pixels with gray level $g$ where $\mathrm{N}_{\mathrm{l}}(\mathrm{g})$ divided by the total number of pixels within the sub-image of size $n \times n(n$ $=9$ ).

$$
P_{l}(g)=\frac{N_{l}(g)}{n^{2}}
$$




$$
\begin{aligned}
& N_{l}(g)=\sum_{i=0}^{n-1} \sum_{j=0}^{n-1} I_{g}(r-1+i, c-1+j) \\
& I_{g}(r, c)=\left\{\begin{array}{l}
1, I(r, c)=g \\
0, I(r, c) \neq g
\end{array}\right\}
\end{aligned}
$$

In order to detect brightness saturation, based on entropy measure as:

$$
I_{\text {satur }}(r, c)=\left\{\begin{aligned}
1, & \text { If } \\
& \left(\text { Entropy }_{0}(\mathrm{r}, \mathrm{c})-\text {EEntropy }_{\mathrm{D}}(\mathrm{r}, \mathrm{c})>\mathrm{Thhr}_{-} \text {satur } 1\right) \\
& \text { And }\left(\text { Entropy }_{0}(\mathrm{r}, \mathrm{c})>\mathrm{Thr} \_ \text {satur2 }\right) \\
0, & \text { Else }
\end{aligned}\right\}
$$

Each pixel is considered as saturation pixel if its activity into distorted image is less than its activity in the original image, or if the original entropy minus distorted entropy for that pixel is more than the saturation threshold Thr_Satur1. It is also ensured that the pixel activity in the original image is more than the saturation threshold Thr Satur2 so that there is enough activity or details to be seen in the original image. The combination of $\mathrm{I}_{\text {noise }}(\mathrm{r}, \mathrm{c})$ and $\mathrm{I}_{\text {satur }}(\mathrm{r}, \mathrm{c})$ are applied with the logical OR as:

$$
I_{\text {noise\&satur }}(r, c)=\sum_{r=0}^{n-1} \sum_{c=0}^{m-1} I_{n o i s e}(r, c) \mid I_{\text {satur }}(r, c)
$$

Finally, the rating $\mathrm{R}$ indicates the result which is the ratio of the total number of pixels with noise artifacts and brightness saturation detected to the total number of pixels.

$$
R=\frac{\sum_{i=0}^{\text {height }-1} \sum_{j=0}^{\text {width }-1} I_{\text {noise\&satur }}(i, j)}{\text { height } \times \text { width }}
$$

\section{Implementation of proposed IQM}

The implementation of IQM was done with Matlab 2012a version. The suggested threshold values were determined empirically to maximize the IQM correlation with MOS of all test images, measured using Pearson Correlation Coefficient (PCC), Root Mean Squared Error (RMSE), Spearman Rank

\begin{tabular}{|c|c|c|c|}
\hline \multirow{4}{*}{$\begin{array}{l}\text { Propose } \\
\text { d IQM }\end{array}$} & Thr_satur1 & \multicolumn{2}{|c|}{1.4} \\
\hline & Thr_satur2 & \multicolumn{2}{|c|}{5.6} \\
\hline & $\begin{array}{c}\text { Thr_Noise_Entr } \\
\text { opy }\end{array}$ & \multicolumn{2}{|c|}{1} \\
\hline & $\begin{array}{c}\text { Standard } \\
\text { thresholds }\end{array}$ & $\begin{array}{c}\text { Original } \\
\text { image } \\
\mathrm{T}=0.019\end{array}$ & $\begin{array}{c}\text { Distorted } \\
\text { image } \\
\mathrm{T}=0.012\end{array}$ \\
\hline $\begin{array}{l}\text { Edge- } \\
\text { based } \\
\text { IQM } \\
\end{array}$ & $\begin{array}{l}\text { Brightness } \\
\text { intensity range } \\
\text { from (0 to 255) }\end{array}$ & $\begin{array}{c}\text { Thr_Low_Lu } \\
\mathrm{m}=30\end{array}$ & $\begin{array}{c}\text { Thr_High_Lum } \\
=250\end{array}$ \\
\hline
\end{tabular}
Order Correlation Coefficient (SROCC), and Outlier Ratio (OR). The implementation is done for the noise artifacts and brightness saturation detection as shown in Table I.

TABLE I. THRESHOLDS VALUES USED INTO IQM IMPLEMENTATION

\section{EVALUATION OF IQM CORRELATION TO HVP}

\section{A. Experiment Setup}

A set of 9 source images from diverse image content was selected from the "Kodak Lossless True Color Image Suite" [15] and LIVE image database [16]. The outcome of 1935 human viewer opinion scores forms a subjective quality assessment in which image quality data were used to assess the IQMs. More details about this experiment can be obtained from [13] and [17].

\section{B. Performance Metrics}

According to [18], the performance of an IQM can be quantitatively evaluated with respect to its ability to predict subjective quality rating in three aspects (Prediction accuracy, Prediction monotonicity, and Predication consistency). The evaluation was done after nonlinear regression for MOS using a five-parameter logistic function to get a better fit for all data. Furthermore, to evaluate the performance of our proposed IQM, current metrics were used for comparison. Along with AMBE and Entropy, Peak Signal to Noise Ratio (PSNR), Multiscale Structural Similarity (MSSIM) and Block-based Spectral Phase Error (BSPE) [19], Information Fidelity Criterion (IFC) based measure [20], and Human Visual System Filtered L2 Norms (HVSL2) [21] were also evaluated. Both MSSIM and IFC are prominent IQMs designed to measure image fidelity in image code. Table II shows the results obtained.

\section{Discussion}

The interpretation of performance metrics [22] shown in Table III explains the discussion of results in Table II.

- AMBE: has poor correlation with MOS. This is agreeing with our review in Section II that brightness change does not always cause annoying effect.

- Entropy: All metrics except SROCC indicate that Entropy has poor correlation with MOS. This indicates that Entropy agrees well with relative magnitude of MOS but fails to accurately predict.

- IFC, HVSL2, PSNR (log), PSPE, and MSSIM: The performance metrics show a fairly relatively correlations to MOS exclude the SROCC of MSSIM which is good over the others.

- Noise IQM: the performance metrics show an excellent correlation to MOS by taking into account the noise artifacts only.

- Proposed IQM: The performance metrics show a higher correlation to MOS by taking into account the brightness saturation with noise artifacts. It clearly outperforms all other IQMs in study.

Also, the execution times were recorded for the proposed IQM, the original IQM and the processes applied (Sobel edge magnitude (EM) and Entropy) where 43 distorted plus 9 source images included into each IQM execution. Table IV shows the 
execution times as measured with Matlab's Tic and Toc functions.

TABLE II. RESULTS OF PERFORMANCE METRICS TO IQMS

\begin{tabular}{|c|c|c|c|c|}
\hline \multirow{2}{*}{ IQMs } & \multicolumn{4}{|c|}{ Performance Metrics } \\
\cline { 2 - 5 } & Pearson CC & $\boldsymbol{R M S E}$ & SROCC & OR \\
\hline AMBA & 0.1346 & 0.7809 & 0.0802 & 0.2791 \\
\hline Entropy & 0.3291 & 0.7510 & 0.7682 & 0.2093 \\
\hline IFC & 0.4025 & 0.7247 & 0.3961 & 0.1860 \\
\hline HVSL2 & 0.5729 & 0.6459 & 0.6284 & 0.1395 \\
\hline PSNR(log) & 0.7009 & 0.5624 & 0.7274 & 0.0930 \\
\hline BSPE & 0.7116 & 0.5536 & 0.7330 & 0.0698 \\
\hline MSSIM & 0.7174 & 0.5490 & 0.7628 & 0.1395 \\
\hline Edge-based IQM & 0.8687 & 0.3964 & 0.8990 & 0 \\
\hline Proposed IQM & 0.8724 & 0.1053 & 0.9225 & 0 \\
\hline
\end{tabular}

TABLE III. PERFORMANCE METRICS INTERPRETATION

\begin{tabular}{|c|c|}
\hline Value of correlation & Interpretation \\
\hline $0.00-0.40$ & Poor \\
\hline $0.41-0.75$ & Fair \\
\hline $0.76-0.85$ & Good \\
\hline $0.86-1.00$ & Excellent \\
\hline
\end{tabular}

TABLE IV. COMPUTATION COMPLEXITY

\begin{tabular}{|c|c|}
\hline IQMs and Important Processes & $\begin{array}{c}\text { Execution Time } \\
(\mathrm{sec})\end{array}$ \\
\hline Edge-based IQM & 128.015 \\
\hline Proposed Enhanced Edge-based IQM & 233.525 \\
\hline $\begin{array}{c}\text { Sobel Edge Magnitude process } \\
\text { (original / distorted image) }\end{array}$ & $0.045 \sim 0.095$ \\
\hline $\begin{array}{c}\text { Entropy process (original / distorted } \\
\text { image) }\end{array}$ & $2.50 \sim 3.40$ \\
\hline
\end{tabular}

\section{CONCLUSIONS}

It is significant for the IQM to correlate well with HVP in order to develop new automated HE-based techniques able to avoid annoying distortions. AMBE and Entropy are found to have poor correlation with HVP. This paper has proposed a generic framework of a new IQM over the two most common distortions and takes into account the significant properties of HVP masking. Evaluation results show that the proposed IQM outperforms significantly the other IQMs in study. Future work recommendation is the reduction of the algorithm's complexity in order to obtain close to real time processing.

\section{REFERENCES}

[1] Y.-T. Kim, "Contrast enhancement using brightness preserving bihistogram equalization", IEEE Transactions on Consumer Electronics, Vol. 43, No.1, pp.1-8, 1997

[2] K. Wongsritong, K. Kittayaruasiriwat, F. Cheevasuvit, K. Dejhan, A. Somboonkaew, "Contrast enhancement using multipeak histogram equalization with brightness preserving", IEEE Asia-Pacific Conference on Circuits and Systems, pp. 455-458, 1998

[3] Y. Wang, Q. Chen, B. Zhang, "Image enhancement based on equal area dualistic sub-image histogram equalization method", IEEE Transactions on Consumer Electronics, Vol. 45, No. 1, pp. 68-75, 1999
[4] S. D. Chen, A. Rahman Ramli, "Preserving brightness in histogram equalization based contrast enhancement techniques", Digital Signal Processing, Vol. 14, No. 5, pp. 413-428, 2004

[5] C. Wang, Z. Ye, "Brightness preserving histogram equalization with maximum entropy: a variational perspective", IEEE Transactions on Consumer Electronics, Vol. 51, No. 4, pp. 1326-1334, 2005

[6] H. Ibrahim, N. S. Pik Kong, "Brightness preserving dynamic histogram equalization for image contrast enhancement", IEEE Transactions on Consumer Electronics, Vol. 53, No. 4, pp. 1752-1758, 2007

[7] D. Menotti, L. Najman, J. Facon, A. De A. Araujo, "Multi-histogram equalization methods for contrast enhancement and brightness preserving", IEEE Transactions on Consumer Electronics, Vol. 53, No. 3, pp. 1186-1194, 2007

[8] M. Kim, M. Gyo Chung, "Recursively separated and weighted histogram equalization for brightness preservation and contrast enhancement", IEEE Transactions on Consumer Electronis, Vol. 54, No. 3, pp. 1389-1397, 2008

[9] C. H. Ooi, N. S .Pik Kong, H. Ibrahim, "Bi-histogram equalization with a plateau limit for digital image enhancement, IEEE Transactions on Consumer Electronis, Vol. 55, No. 4, pp. 2072-2080, 2009

[10] C. H. Ooi, N. A. M. Isa, "Adaptive contrast enhancement methods with brightness preserving, IEEE Transactions on Consumer Electronis", Vol. 56, No. 4, pp. 2543-2551, 2010

[11] S.-H. Yun, J. H. Kim, S. Kim, "Image enhancement using a fusion framework of histogram equalization and Laplacian pyramid", IEEE Transactions on Consumer Electronis, Vol. 56, No. 4, pp. 2763-2771, 2010

[12] N. Sengee, A. Sengee, H. K. Choi, "Image contrast enhancement using bi-histogram equalization with neighborhood metrics", IEEE Transactions on Consumer Electronics, Vol. 56, No. 4, pp. 2727-2734, 2010

[13] S. D. Chen, "A new image quality measure for assessment of histogram equalization-based contrast enhancement techniques", Digital Signal Processing, Vol. 22, No. 4, pp. 640-647, 2012

[14] S. D. Chen, Y. Al-Najja,N. H. Azami, K. S. Beh, "Measuring Image Quality for Assessment of Contrast Enhancement Techniques", Australian Journal of Basic and Applied Sciences, Vol. 7, No. 11, pp. 178-188, 2013

[15] Rich Franzen, Kodak lossless true color image suite, http://r0k.us/graphics/kodak/

[16] H. R. Sheikh, Z. Wang, L. Cormack, A. C. Bovik, LIVE image quality assessment database release 2, http://live.ece.utexas.edu/research/ quality

[17] S. D. Chen, M. Singh, "Re-evaluation of automatic global histogram equalization-based contrast enhancement methods", Electronic Journal of Computer Science and Information Technology, Vol. 1, No.1, 2011

[18] VQEG, VQEG final report of FR-TV phase II validation test, 2003

[19] Z. Wang, A. C. Bovik, H. R. Sheikh, E. P. Simoncelli, "Image quality assessment:from error visibility to structural similarity", IEEE Transactions on Image Processing, Vol. 13, No. 4, pp. 600-612, 2004

[20] H. R. Sheikh, A. C. Bovik, G. de Veciana, "An information fidelity criterion for image quality assessment using natural scene statistics", IEEE Transactions on Image Processing, Vol. 14, No. 12, pp. 21172128,2005

[21] I. Avcibas, B. Sankur, K. Sayood, "Statistical evaluation of image quality measures",Journal of Electronic Imaging, Vol. 11, No. 2, pp. 206-223, 2002

[22] H. Arsham, Statistical thinking for managerial decisions, Available at: http://home.ubalt.edu/ntsbarsh/Business-stat/opre504.htm, 2014 